\title{
Estandarización del método propuesto por la Organización Mundial de la Salud para determinar los niveles de susceptibilidad de los vectores de leishmaniasis a insecticidas
}

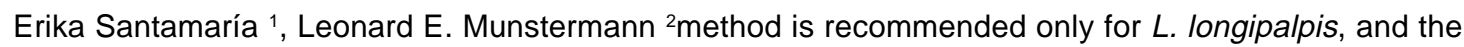
modified method for $L$. serrana, $L$. quasitownsendi and closely related species.

El objetivo de este estudio fue estandarizar el método OMS para determinar susceptibilidad a insecticidas en algunas especies de Lutzomyia en condiciones de laboratorio y de campo. Los ensayos biológicos se realizaron sin insecticida, únicamente con tratamientos controles para determinar mortalidad por manipulación y otras condiciones desfavorables para cada especie. Se emplearon hembras de tres colonias de laboratorio: dos cepas de Lutzomyia longipalpis y una de Lutzomyia serrana. También se incluyeron en el estudio hembras silvestres de Lutzomyia quasitownsendi. Se emplearon dos tipos de métodos: el método OMS y el método OMSadaptado; este último consistió en reemplazar el tubo OMS para el periodo posexposición por un contenedor plástico con yeso humedecido. Para cada método se ensayaron tres tipos de papeles: papel impregnado con aceite de oliva, papel impregnado con aceite de silicona y papel sin aditivos. La variable por medir fue el porcentaje de mortalidad a las $24 \mathrm{~h}$. El método OMS resultó conveniente para las dos cepas de L. longipalpis con una mortalidad entre 0 y $10 \%$, pero registró una mortalidad elevada en L. serrana (20-80\%) y L. quasitownsendi silvestres (10-50\%). Con el método OMS-modificado, la mortalidad promedio fue inferior a $4 \%$ en todas las especies ensayadas. Con respecto al tipo de papel, no se encontraron variaciones importantes. Según los resultados, el recipiente utilizado en el periodo posexposición afecta la supervivencia de los flebótomos y de manera variable para cada especie. Al establecer líneas base de susceptibilidad a insecticidas en especies del género Lutzomyia, se recomienda el uso del método OMS para L. longipalpis y del método OMS-modificado para $L$. serrana, $L$. quasitownsendi y especies cercanas.

Palabras clave: Lutzomyia, susceptibilidad a insecticidas, ensayos biológicos.

Standardization of WHO-proposed method for determining susceptibility levels of leishmaniasis vectors to insecticides

The WHO method for determining insecticide resistance was standardized for several species of Lutzomyia sand flies under laboratory and field conditions. The biological assays were applied solely to optimize the conditions for the control, i.e., without insecticide, and to estimate mortality due to handling or other unfavorable conditions. Adult female flies from 3 laboratory colonies and one field strain were tested: two laboratory strains of Lutzomyia longipalpis, one laboratory strain of Lutzomyia serrana and one field-collected strain of Lutzomyia quasitownsendi. The WHO method was compared with one modified in which, during the post-exposure period, the recommended plain tube apparatus was replaced with a plastic container layered with damp plaster of Paris. Three paper substrate types were compared under each condition: olive oil additive, silicon oil additive and plain paper. The measured variable was percent mortality in 24 h. For the WHO protocol, the L. longipalpis strains indicated a 0-10\% mortality, L. serrana 20$80 \%$ and $L$. quasitownsendi $10-50 \%$. With the modified WHO apparatus, the average mortality was $<4 \%$ for all species. No significant differences were observed among the paper treatments. These results indicate a strong species-specific effect of post-exposure conditions on sand flies. To establish baseline levels of insecticide resistance in Lutzomyia sand flies, the WHO 
method is recommended only for $L$. longipalpis, and the modified method for $L$. serrana, $L$. quasitownsendi and closely related species.

Key words: Lutzomyia, insecticide susceptibility, bioassays.

El control de las leishmaniasis contempla entre sus posibilidades el uso de insecticidas que disminuyan la densidad de los vectores por debajo de los niveles críticos para la transmisión, siendo especialmente importante en situaciones en las que los insectos frecuentan las viviendas y sus alrededores. Actualmente, el control químico suscita gran interés debido a la progresiva adaptación de las especies de flebótomos al domicilio y a los recientes registros de casos urbanos de la enfermedad, en su forma cutánea y visceral, en Venezuela, Brasil y Colombia (1-4).

Para el control de los flebótomos en el domicilio y peridomicilio, insecticidas como el DDT, el malatión, el fenitrotión, el propoxur y los piretroides sintéticos se han usado con relativo éxito en varios países (5). Sin embargo, como resultado de la presión de los insecticidas empleados en agricultura y campañas antivectoriales, las poblaciones de flebótomos pueden verse afectadas y desarrollar resistencia (6). Por esta razón, en cualquier programa de control de vectores es importante conocer la susceptibilidad a insecticidas de las especies objeto del control y revisar la susceptibilidad después de cada intervención. Con la vigilancia adecuada, se realiza un seguimiento de la tolerancia de las especies a los insecticidas y se detectan oportunamente signos de resistencia.

El método convencional para determinar el nivel inicial de susceptibilidad de los flebótomos a los insecticidas es el propuesto por la Organización Mundial de la Salud (OMS) y es el mismo empleado para mosquitos adultos desde la década de los sesenta. De manera abreviada, el método consiste en exponer grupos de insectos a papeles impregnados con diferentes dosis de insecticidas por un periodo de tiempo definido y dejarlos en ausencia del insecticida por 24 horas. Con los Correspondencia:

Cristina Ferro

mferro@hemagogus.ins.gov.cogus.ins.gov.co

Recibido: 12/02/02; aceptado: 30/05/02 resultados, se construye una línea de regresión concentración-mortalidad y se determinan los parámetros estadísticos $\mathrm{CL}_{50}$ y $\mathrm{CL}_{95}$ (concentraciones de insecticida que producen una mortalidad del 50 y del $95 \%$, respectivamente). De igual forma, las pruebas pueden realizarse con una única concentración discriminante o diagnóstica empleando diferentes tiempos de exposición, determinando $\mathrm{TL}_{50} \mathrm{y} \mathrm{TL}_{95}$ (tiempos de exposición necesarios para producir, respectivamente, una mortalidad del 50 y del $95 \%$ ). Siempre se debe incluir un control (o testigo) por cada insecticida probado y si la mortalidad en este grupo excede el $20 \%$, la prueba debe descartarse $(7,8)$.

Aunque existe buena documentación sobre la susceptibilidad y el estado de resistencia a insecticidas de muchas especies de insectos de importancia médica, especialmente de los géneros Aedes, Culex y Anopheles (9-13), pocos estudios se han informado sobre las líneas base de susceptibilidad de los flebótomos a los insecticidas. En el Viejo Mundo son relativamente escasos los trabajos en este tópico (14-18) y en el Nuevo Mundo se ha determinado la susceptibilidad a insecticidas de sólo dos especies de Lutzomyia: Lutzomyia youngi Feliciangeli y Murillo 1985 (19-21) y Lutzomyia longipalpis (Lutz y Neiva 1912) (6,22-24). En casi todos los casos, el método empleado presenta modificaciones en la metodología propuesta por la OMS, lo cual dificulta la comparación e interpretación de la información.

Se han atribuido varias limitaciones y problemas técnicos al método OMS y su estuche de materiales, como son: el costo, las dificultades de envío, los papeles impregnados con una sola dosis de cada insecticida, el requerimiento de un gran número de mosquitos de la misma población y las dificultades para utilizar en el campo (25). Específicamente para flebótomos, en uno de los trabajos informan que el equipo OMS presenta inconveniencias, ya que los insectos (L. youngi) escapan a través de los dispositivos de 
intercambio entre el tubo de exposición y el de observación (19).

En los ensayos preliminares con el estuche de materiales y el procedimiento OMS para determinar la susceptibilidad a insecticidas en algunas especies de Lutzomyia, observamos alta mortalidad en los controles, presumiblemente causada por las condiciones del periodo posexposición, por lo cual se decidió ensayar un contenedor plástico con yeso humedecido como recipiente alternativo para este periodo.

El objetivo de este estudio fue estandarizar el procedimiento OMS para tres especies de Lutzomyia, comparando la mortalidad obtenida en los controles (sin insecticida) con ambos procedimientos: 1) siguiendo fielmente el método OMS y 2) adicionando la modificación propuesta.

\section{Materiales y métodos}

Especies de Lutzomyia utilizadas: se emplearon hembras de Lutzomyia de uno a tres días después de la emergencia de tres colonias de laboratorio: $L$. longipalpis $\left(\mathrm{F}_{51}\right.$, proveniente de El Callejón, Cundinamarca), L. longipalpis $\left(\mathrm{F}_{13}\right.$, proveniente de Coyaima, Tolima) y $L$. serrana (Damasceno y Arouck) $\left(F_{17}\right.$, proveniente de Tuluá, Valle). Los métodos para el establecimiento y mantenimiento de las colonias de Lutzomyia se describen en Modi y Tesh (26) y Ferro et al. (27).

Adicionalmente, se efectuaron ensayos en el campo en un foco de leishmaniasis cutánea en Bituima (Cundinamarca) con L. quasitownsendi Osorno, Osorno-Mesa \& Morales, 1972, el flebótomo más abundante en esta zona (datos sin publicar, Unidad Básica de Entomología, Laboratorio de Salud Pública de Cundinamarca).

Las hembras empleadas en los ensayos biológicos no habían sido alimentadas con sangre, ya que en ensayos preliminares se observó una mayor fragilidad y mortalidad de las hembras con alimento sanguíneo frente a hembras sin este alimento. Por otro lado, en la mayoría de los estudios de susceptibilidad a insecticidas en flebótomos se utilizan hembras sin alimentación sanguínea (6,15-17).

Equipo y papeles impregnados suministrados por la OMS: cada prueba, como describe la OMS (7), se realizó con dos tubos plásticos, uno de observación y otro de exposición. Los dos tubos son iguales. Por medio de un círculo de $0,6 \mathrm{~cm}$ de diámetro de color verde o rojo, ubicado lateralmente en el exterior de cada tubo, se pueden diferenciar los tubos entre ellos. El de color verde corresponde al tubo de observación y el de color rojo al de exposición. Los dos tubos se conectan entre sí por medio de una unión, la cual se enrosca a uno de los extremos de cada uno de los tubos mencionados. Cuando se requiere, esta unión permite, a través de un sistema corredizo, el paso de los insectos de un tubo al otro. Para las pruebas, cada tubo fue recubierto con una hoja de papel enrollada en forma de cilindro. En el tubo de observación se colocó papel bond blanco y en el tubo de exposición, la hoja de papel a probar. Estas hojas se sujetaron a los extremos de los tubos respectivos con un resorte anular, el cual en el tubo de observación era de plata y en el de exposición, de cobre.

Se emplearon papeles impregnados con aceite de oliva y aceite de silicona (generalmente utilizados como controles para insecticidas organofosforados y piretroides, respectivamente). Los aceites son el medio que transporta el ingrediente activo del insecticida y lo distribuye en el papel. Los papeles consisten en rectángulos de papel filtro Whatman No. 1 de $12 \times 15 \mathrm{~cm}$, impregnados con el medio (aceite) correspondiente a una concentración de $3,6 \mathrm{mg} / \mathrm{cm}^{2}$.

Ensayos biológicos siguiendo el método OMS y el método OMS-modificado: las hembras a utilizar en los ensayos se separaron por especie en una jaula de muselina. Las condiciones de humedad se mantuvieron introduciendo la jaula con los insectos en una bolsa plástica oscura, la cual permitía el paso de la luz de forma atenuada. De esta forma se mantienen habitualmente los ejemplares adultos en las colonias de laboratorio. Antes de realizar las pruebas, la jaula dentro de la bolsa fue trasladada a un laboratorio contiguo, donde se realizaron los ensayos. Con el fin de tener un periodo de adaptación de las hembras a la iluminación del cuarto, se retiró la bolsa plástica oscura de 15 a 20 min antes de comenzar las pruebas. Se registraron la temperatura y la humedad del lugar. 
El procedimiento OMS, tal y como lo describe el protocolo (7), se dividió en tres periodos: 1) preexposición: con el aspirador de boca se tomaron de la jaula grupos de 9 a 12 hembras y se ubicaron en el tubo de observación, donde permanecieron por espacio de 15 a $20 \mathrm{~min}$; 2) exposición: las hembras se trasladaron al tubo de exposición con el tratamiento correspondiente; el extremo del tubo se orientó hacia la fuente de luz de forma tal que la mayoría de las hembras pasaron por fototropismo y las restantes fueron sopladas con suavidad. Estos tubos se ubicaron verticalmente y con el tamiz hacia arriba durante una hora; 3) posexposición: los insectos se trasladaron nuevamente al tubo de observación donde permanecieron 24 horas, con suplemento de agua y solución azucarada. Pasado este tiempo, se registró la mortalidad. Este procedimiento se denominará en adelante método OMS.

La adaptación al procedimiento consistió en reemplazar el tubo OMS para el periodo posexposición por un contenedor plástico $(5,5 \mathrm{~cm}$ de alto y $6,6 \mathrm{~cm}$ de diámetro) que en el fondo contiene una capa de yeso dental de $1,5 \mathrm{~cm}$ de grosor y en la parte superior una malla de muselina ajustada al contenedor con una banda elástica. Este recipiente es descrito en detalle por Ferro et al. (27) y Santamaría et al. (28). El yeso sirve como medio para mantener la humedad dentro del recipiente. En condiciones de campo, en horas nocturnas, la humedad relativa fue alta (entre 70 y $80 \%$ ) y el yeso en el recipiente absorbió la humedad del ambiente. En las condiciones del laboratorio en Bogotá, donde la humedad relativa es cercana al $50 \%$, fue necesario adicionar 8 a $10 \mathrm{ml}$ de agua al yeso unos minutos antes de usar el recipiente. Los pasos a seguir son similares al método OMS, pero después de haber pasado las hembras al tubo de observación para el periodo posexposición, las hembras fueron transferidas suavemente con aspirador de boca a los contenedores plásticos, donde permanecieron 24 horas. Cumplido este tiempo, se realizó la lectura de la mortalidad. Este procedimiento se denominará en adelante método OMS-adaptado (figura 1).

En los dos métodos, los recipientes permanecieron durante el periodo de exposición

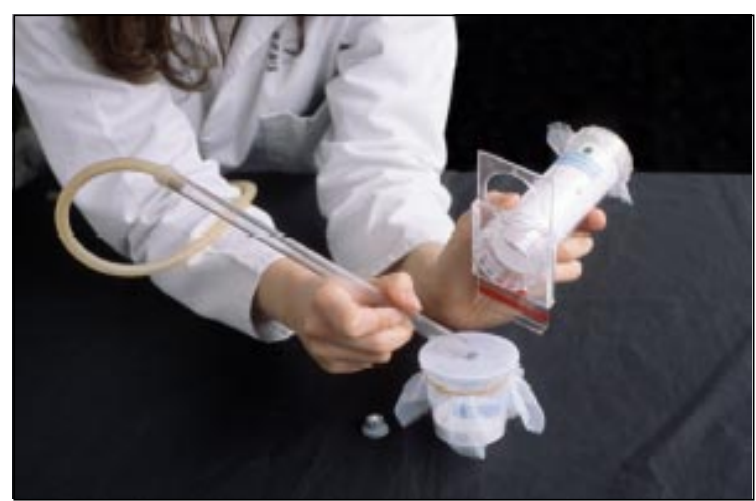

Figura 1. Traslado de los insectos desde el tubo de observación OMS al recipiente posexposición OMSadaptado.

y posexposición en una caja de poliestireno (icopor) con una gasa humedecida, termómetro de máximas y mínimas e higrómetro. Durante el periodo posexposición, las hembras tenían acceso a motas de algodón embebidas en agua y solución azucarada ubicadas en la parte superior de los recipientes.

Los tipos de papel a probar con los dos métodos fueron: 1) papel impregnado con aceite de oliva y 2) papel impregnado con aceite de silicona. Como control (Control-1), se empleó papel filtro sin adición de medio (aceites). La variable a medir fue el porcentaje de mortalidad a las 24 horas. Se realizaron cinco réplicas de cada tipo de papel para cada especie.

Adicionalmente, para $L$. serrana se incluyó otro control (Control-2), que consistió en dejar grupos de 10 hembras en los dos contenedores ensayados para el periodo posexposición (tubo de observación-OMS y contenedor con yeso humedecido) sin aplicar ningún método. Pasadas 24 horas, se realizó la lectura de mortalidad. Se realizaron cinco réplicas para cada contenedor.

En el análisis de datos se realizó un análisis de varianza de dos factores para determinar si había diferencias en la mortalidad de las especies por efecto del método (OMS y OMS-modificado) y el tipo de papel (impregnado con aceite de oliva y silicona).

\section{Resultados}

En el cuadro 1 se presentan los porcentajes de mortalidad promedio para cada especie según el 
Cuadro 1. Efecto general del método sobre la mortalidad de L. longipalpis, L. serrana y L. quasitowsendi.

\begin{tabular}{llrrrrr}
\hline \multirow{2}{*}{ Especie } & \multicolumn{1}{c}{ Método } & $\mathbf{n}$ & $\begin{array}{c}\text { \%Mortalidad } \\
\text { promedio }\end{array}$ & $\mathbf{D E}^{2}$ & & \multicolumn{2}{c}{ Valores } \\
\cline { 5 - 7 } & & & & mínimo & máximo \\
\hline L. longipalpis & OMS & 18 & 1,1 & 3,08 & 0,0 & 10,0 \\
& OMS-ADAPTADO & 18 & 0 & 0 & 0 & 0 \\
L. serrana & OMS & 15 & 43,7 & 20,7 & 20,0 & 80,0 \\
& OMS-ADAPTADO & 15 & 1,3 & 3,3 & 0 & 10,0 \\
L. quasitownsendi & OMS & 15 & 27,2 & 13,6 & 10,0 & 40,0 \\
& OMS-ADAPTADO & 15 & 4,0 & 5,2 & 0 & 10,0 \\
\hline
\end{tabular}

${ }^{1}$ Número de réplicas (9-11 hembras/réplica)

${ }^{2}$ Desviación estándar

método. Los resultados de las dos cepas de laboratorio de L. longipalpis (Callejón $\mathrm{F}_{51}$ y Coyaima $F_{13}$ ) fueron muy similares, por lo cual se tomaron de manera conjunta. Esta especie no se vio afectada por ninguno de los dos métodos, presentando porcentajes de mortalidad por debajo del 10\% (considerada como normal). En contraste, las otras especies presentaron elevados porcentajes de mortalidad por el método OMS, siendo la mortalidad promedio de $L$. serrana mayor que la de L. quasitownsendi $(43,7$ y $27,2 \%$, respectivamente); mientras que con el método OMS-adaptado, las mortalidades fueron mínimas (1,3 y 4,0\%, respectivamente). Esta diferencia debida al método fue estadísticamente significativa en el análisis de varianza de dos factores tanto para $L$. serrana $(F=90,6 ; g l=1,24$; $P<0,001)$, como para $L$. quasitownsendi $(F=53,6$; $\mathrm{gl}=1,24 ; P<0,001)$.

En el cuadro 2 se presenta la mortalidad con relación al tipo de papel empleado; no se incluyó L. longipalpis, ya que presentó porcentajes de mortalidad de $0 \%$ en la mayoría de los casos. Para L. serrana y L. quasitownsendi no se encontraron diferencias significativas en ninguna de las dos especies por efecto del tipo de papel utilizado.

Con respecto al Control 2, realizado únicamente con ejemplares de $L$. serrana criados en laboratorio, se encontró que al dejar las hembras en el tubo de observación OMS por 24 horas sin ningún traslado, presentaron un porcentaje de mortalidad promedio de $48,7 \%(33,3-60 \%$, $\mathrm{DE}=11,9)$, mientras que las hembras dejadas en las mismas condiciones en los contenedores con yeso humedecido no presentaron mortalidad en ninguna de las cinco réplicas.

Las condiciones ambientales en los periodos de exposición y posexposición fueron: en laboratorio (temperatura $=22-24{ }^{\circ} \mathrm{C}$ y $\mathrm{HR}=80-90 \%$ ) y en campo (temperatura $=19-22{ }^{\circ} \mathrm{C}$ y $\mathrm{HR}=85-90 \%$ ).

\section{Discusión}

La información sobre el uso del método estándar de la OMS para determinar susceptibilidadresistencia a insecticidas en vectores de leishmaniasis es muy limitada y los pocos trabajos realizados incluyen modificaciones sustanciales que dificultan la comparación de los resultados, como variar la duración del periodo de exposición y posexposición, utilizar hembras alimentadas o mezclas de hembras y machos $(19,24)$. Por este motivo, se hace necesario proponer un método unificado que se adapte a las diferentes especies de flebótomos de importancia epidemiológica en nuestro país y otras regiones similares.

Siguiendo el método OMS, se presentaron variaciones importantes entre especies. Por un lado, las dos cepas de $L$. longipalpis respondieron bien al método, con mortalidades de $0 \%$ en la mayoría de los casos. Para L. serrana, en cambio, los porcentajes de mortalidad oscilaron entre $20 \mathrm{y}$ $80 \%$ y para $L$. quasitownsendi silvestres entre el 10 y el $50 \%$.

Estos elevados porcentajes de mortalidad llevaron a buscar alternativas sin cambiar las líneas generales del método OMS. Se decidió, entonces, ensayar para el periodo de posexposición (24 h) un recipiente diferente al tubo OMS. El contenedor plástico ensayado en el método OMS-adaptado 
Cuadro 2. Efecto del método y tipo de papel sobre la mortalidad de L. serrana y L. quasitownsendi.

\begin{tabular}{|c|c|c|c|c|c|c|}
\hline \multirow[b]{2}{*}{ Especie } & \multirow[b]{2}{*}{ Método } & \multirow{2}{*}{$\begin{array}{l}\text { Tipo de } \\
\text { papel }\end{array}$} & \multirow{2}{*}{$\begin{array}{c}\text { \%Mortalidad } \\
\text { promedio }^{1}\end{array}$} & \multirow[b]{2}{*}{ DE } & \multicolumn{2}{|c|}{ Valores } \\
\hline & & & & & mínimo & máximo \\
\hline \multirow[t]{6}{*}{ L. serrana } & OMS & Ac. oliva & 34,0 & 11,0 & 20,0 & 50,0 \\
\hline & & Ac. silicona & 50,0 & 18,7 & 30,0 & 80,0 \\
\hline & & Control 1 & 47,1 & 19,2 & 20,01 & 70,0 \\
\hline & OMS-ADAPTADO & Ac. oliva & 2,0 & 4,47 & 0 & 10 \\
\hline & & Ac. silicona & 2,0 & 4,47 & 0 & 10 \\
\hline & & Control 1 & 0 & 0 & 0 & 0 \\
\hline \multirow[t]{6}{*}{ L. quasitownsendi } & OMS & Ac. oliva & 40,0 & 7,1 & 30,0 & 50,0 \\
\hline & & Ac. silicona & 22,7 & 13,6 & 10,0 & 40,0 \\
\hline & & Control 1 & 18,9 & 11,7 & 10,0 & 33,3 \\
\hline & OMS-ADAPTADO & Ac. oliva & 6,0 & 5,6 & 0 & 11,1 \\
\hline & & Ac. silicona & 1,8 & 4,1 & 0 & 9,1 \\
\hline & & Control 1 & 4,2 & 5,8 & 0 & 11,1 \\
\hline
\end{tabular}

${ }^{15}$ réplicas/tipo de papel (9-11 hembras/réplica)

es el recipiente en el que generalmente se mantienen los flebótomos en las colonias de laboratorio en todas las fases de su ciclo de vida $(26,29)$. Con esta adaptación, los porcentajes de mortalidad fueron mínimos tanto para L. serrana como para L. quasitownsendi.

Los resultados de los ensayos denominados Control-2 (mantenimiento de los insectos en los contenedores sin ningún tratamiento por 24h), implican directamente al contenedor como el responsable de la alta mortalidad en el métodoOMS. La explicación probablemente está en las condiciones microclimáticas de los recipientes, siendo la humedad la diferencia más importante, pues en el contenedor del método OMS-adaptado ésta se mantiene elevada por la presencia de una base de yeso que permanece humedecida, condición ausente en el tubo OMS.

Sin embargo, los requerimientos de humedad relativa en este tipo de ensayos biológicos no son similares para todas las especies de Lutzomyia. En los ensayos con L. longipalpis, se observó que las hembras no se afectaron por la permanencia en el tubo-OMS. L. longipalpis es una especie relativamente robusta en comparación con la mayoría de especies del genero Lutzomyia que son delicadas y de tamaño pequeño (30). L. longipalpis se puede encontrar en una gran diversidad de hábitats con amplios márgenes de humedad relativa $(31,32)$. Estas características probablemente le permitieron soportar la manipulación y el reposo sobre el papel sin mayores contratiempos. Por otro lado, $L$. serrana, es una especie de menor tamaño encontrada generalmente en bosques tropicales húmedos y muy húmedos $(33,34)$, lo que puede explicar la muerte después del contacto prolongado con el papel y las superficies del tubo-OMS.

Con respecto a los tipos de papel utilizados (impregnados con aceite de oliva, aceite de silicona y control), no se presentaron variaciones importantes y no se demostró mayor porcentaje de mortalidad en algún tipo de papel específico.

En cuanto a la mortalidad en los testigos (controles), el procedimiento OMS establece que: "Las pruebas que acusen una mortalidad superior al $20 \%$ en los testigos no se considerarán válidas y deberán repetirse; si la mortalidad de los testigos está comprendida entre el 5 y el $20 \%$, se recomienda corregir los porcentajes de mortalidad con la fórmula Abbott (\%mortalidad de la prueba-\%mortalidad de testigos $\times 100 / 100-\%$ mortalidad de testigos) y si la mortalidad de los testigos es menor del $5 \%$ es considerada como normal y no se practica corrección a los datos" (7). Según esto, al seguir el método OMS, las pruebas realizadas con $L$. 
longipalpis se considerarían válidas, mientras que las pruebas con $L$. serrana y $L$. quasitownsendi deberían descartarse. Con el método OMSadaptado, se obtienen mortalidades de muy bajas a nulas en los controles; además, la adaptación incluida no cambia sustancialmente el método original, ya que simplemente se reemplaza el recipiente de observación y no se alteran los tiempos establecidos de exposición y posexposición.

Se recomienda, previo a la determinación de susceptibilidad a insecticidas en vectores de leishmaniasis, ajustar el procedimiento a utilizar. Esto para confirmar que los insectos mueren por la acción de la sustancia tóxica y no por la manipulación y condiciones en las que se realizan los ensayos.

En conclusión, el método OMS fue conveniente para la especie $L$. longipalpis y la adaptación realizada al método fue favorable para $L$. serrana (en condiciones de laboratorio) y para $L$. quasitownsendi (en condiciones naturales). Teniendo en cuenta que la mayoría de especies de Lutzomyia de importancia médica en las Américas presentan características similares a $L$. serrana y $L$. quasitownsendi, podría recomendarse el método OMS-adaptado para determinar susceptibilidad a insecticidas en las especies de Lutzomyia del Nuevo Mundo.

\section{Agradecimientos}

Por la financiación, a la Organización Panamericana de la Salud (ASC-99/00099-1), al proyecto Genetics and Biogeography of Sand Fly Disease Vectors (Al-34521) y al Instituto Nacional de Salud (20003100302). A Colciencias por su apoyo a grupos de investigación (convocatoria año 2000, contrato 338). A Raúl H. Pardo por su asesoría en el análisis estadístico; a Patricia Fuya por su colaboración en los ensayos con ejemplares silvestres y a Marco Fidel Suárez por el mantenimiento de las colonias de laboratorio.

\section{Referencias}

1. Aguilar CM, Fernández R, Fernández E, Cannova DC, Ferrer E, Cabrera Z, et al. Urban visceral leishmaniasis in Venezuela. Mem Inst Oswaldo Cruz 1998;93:15-6.
2. Oliveira AM, Melo MT. Vectors control importance on leishmaniasis transmission. Mem Inst Oswaldo Cruz 1994;89:451-6.

3. Pardo R, Farieta S, Munstermann LE, Ferro C. Estudio preliminar de los flebótomos de Villeta y Quebradanegra, Cundinamarca: sus implicaciones en salud pública. Biomédica 1996;16:293-302.

4. Sandoval CM, Angulo VM, Gutiérrez R, Muñoz G, Ferro C. Especies de Lutzomyia (Diptera: Psychodidae) posibles vectores de leishmaniasis en la ciudad de Bucaramanga, Santander, Colombia. Biomédica 1998; 18:161-8.

5. World Health Organization. Control of the leishmaniases. Report of a WHO Expert Committee. Technical Report Series. Geneva: WHO; 1990.

6. Mazzarri MB, Feliciangeli MD, Maroli M, Hernández A, Bravo A. Susceptibility of Lutzomyia longipalpis (Diptera: Psychodidae) to selected insecticides in an endemic focus of visceral leishmaniasis in Venezuela. J Am Mosq Control Assoc 1997;13:335-41.

7. Organización Mundial de la Salud. Resistencia a los insecticidas y lucha contra vectores. Serie de informes técnicos N.443. $17^{\circ}$ Informe del Comité de Expertos de la OMS en Insecticidas. Ginebra: OMS; 1970.

8. World Health Organization. Instructions for determining the susceptibility or resistance of adult mosquitoes to organochlorine, organophosphate and carbamate insecticides. Establishment of the baseline. 1981. WHO/ VBC/81.805.

9. Suárez MF, Morales CA. Pruebas de susceptibilidad de Aedes aegypti al ciflutrin en Colombia. Salud Pública 2000;15:109-13.

10. Rodríguez MM, Bisset J, Rodríguez I, Días C. Determinación de la resistencia a insecticidas y sus mecanismos bioquímicos en 2 cepas de Culex quinquefasciatus procedentes de Santiago de Cuba. Rev Cubana Med Trop 1997;49:209-14.

11. Ocampo CG, Brogdon WG, Orrego CM, Toro G, Montoya-Lerma J. Insecticide susceptibility in Anopheles pseudopunctipennis from Colombia: comparison between bioassays and biochemical assays. J Am Mosq Control Assoc 2000;16:331-8.

12. Wang J. Resistance to two pyrethroids in Anopheles sinensis from Zhejiang, China. J Am Mosq Control Assoc 1999;15:308-11.

13. Mazzarri M, Georghiou G. Characterization of resistance to organophosphate, carbamate, and pyrethroid insecticides in field population of Aedes aegypti from Venezuela. J Am Mosq Control Assoc 1995;11:315-22.

14. Amalraj DD, Sivagnaname N, Srinivasan $\mathbf{R}$. Susceptibility of Phlebotomus argentipes and $P$. papatasi 
(Diptera: Psychodidae) to insecticides. J Commun Dis 1999;31:177-80.

15. Seyedi MA, Yezdan H, Shah H, Jeradi M. Susceptibility of Phlebotomus papatasi (Diptera: Psychodidae) to DDT in some foci of cutaneous leishmaniasis in Iran. J Am Mosq Control Assoc 1992;8:99-100.

16. Fahmy AR, Khater EIM, EI Sawaf B, Shehata M. Insecticide susceptibility status of field population of sandfly Phlebotomus papatasi in the Sinai Peninsula, Egypt. Geneva: WHO; 1996. p.1-9.

17. Tetreault GE, EI-Baset A, Hanafi AH, Beavers GM, Zeichner BC. Susceptibility of sand flies to selected insecticides in North Africa and the Middle East. J Am Mosq Control Assoc 2001;17:23-7.

18. Kaul SM, Wattal BL, Bhatnagar VN, Mathur KK. Preliminary observations on the susceptibility status of Phlebotomus argentipes and $P$. papatasi to DDT in two districts of North Bihar (India). J Commun Dis 1978;10: 208-11.

19. Scorza JV, Márquez M. Control de Lutzomyia young en el área endémica venezolana de leishmaniasis tegumentaria. Bol Dir Mal San Amb 1989;29:35-41.

20. Scorza JV, Rosario CI, Scorza-D JV, Rojas E. Susceptibilidad de hembras silvestres de Lutzomyia youngi de Trujillo, Venezuela, a insecticidas sintéticos. Bol Dir Mal San Amb 1995;35(Suppl1):311-26.

21. Rojas E, Alvarez L, Scorza JV. A practical device for testing sand flies with insecticides. III International Symposium on Phlebotomine Sandflies, Montpellier, Francia, agosto de 1999

22. Rocha A, Texeira C, Ferreira CM. Susceptibility of Lutzomyia longipalpis to deltamethrin. Mem Inst Oswaldo Cruz 1988;83:395-6.

23. Quesada BL, Montoya J. Evaluación de insecticidas sobre flebotomineos Lutzomyia en condiciones de laboratorio. Biomédica 1991;11(Suppl1):88-9.

24. Oliveira AM, Melo MT. Sandflies susceptibility to insecticides of the four main chemical groups. Rev Soc Bras Med Trop 1994;27(Suppl1):356.
25. Brogdon W. Biochemical resistance detection: an alternative to bioassay. Parasitology Today 1989;5:56-60.

26. Modi GB, Tesh RB. A simple technique for mass rearing Lutzomyia longipalpis and Phlebotomus papatasi (Diptera: Psychodidae) in the laboratory. J Med Entomol 1983;20:568-9.

27. Ferro C, Cárdenas E, Corredor D, Morales A, Munstermann LE. Life cycle and fecundity analysis of Lutzomyia shannoni (Dyar) (Diptera: Psychodidae). Mem Inst Oswaldo Cruz 1998;93:195-9.

28. Santamaría E, Munstermann LE, Ferro C. Estimating carrying capacity in a newly colonized sand fly Lutzomyia serrana (Diptera: Psychodidae) colony. J Econ Entomol 2002;95:149-54.

29. Endris RG, Young DG, Butler JF. The laboratory biology of the sand fly Lutzomyia anthophora (Diptera: Psychodidae). J Med Entomol 1984;21:656-64.

30. Killick-Kendrick R, Leaney AJ, Ready PD. The establishment, maintenance and productivity of a laboratory colony of Lutzomyia longipalpis (Diptera: Psychodidae). J Med Entomol 1977;13:429-40.

31. Young DG, Duncan MA. Guide to the identification and geographic distribution of Lutzomyia sand flies in México, West Indies, Central and South America (Diptera: Psychodidae). Mem Am Entomol Inst 1994; 54:1-881.

32. Ferro C, Morrison AC, Torres M, Pardo R, Wilson ML, Tesh RB. Species composition and relative abundance of sand flies of the genus Lutzomyia (Diptera: Psychodidae) at an endemic focus of visceral leishmaniasis in Colombia. J Med Entomol 1995;32:52737.

33. Travi BL, Montoya J, Solarte Y, Lozano L, Jaramillo C. Leishmaniasis in Colombia. 1. Studies on the phlebotomine fauna associated with endemic foci in the Pacific coast region. Am J Trop Med Hyg 1988;39:261-6.

34. Alexander B, Ferro C, Young DG, Morales A, Tesh R. Ecology of phlebotomine sand flies (Diptera: Psychodidae) in a focus of Leishmania (Viannia) braziliensis in northeastern Colombia. Mem Inst Oswaldo Cruz 1992;87:387-95. 